\title{
Semi-supervised Learning of Classifiers: Theory, Algorithms and Their Application to Human-Computer Interaction
}

Ira Cohen, Fabio G. Cozman, Nicu Sebe, Marcelo C. Cirelo, Thomas S. Huang

Ira Cohen: Hewlett-Packard Labs, Palo Alto, CA, USA, ira.cohen@hp.com

Fabio G. Cozman and Marcelo C. Cirelo: Escola Politécnica, Universidade de São Paulo, São Paulo,Brazil. fgcozman@usp.br, marcelo.cirelo@poli.usp.br

Nicu Sebe: Faculty of Science, University of Amsterdam, The Netherlands. nicu@ science.uva.nl

Thomas S. Huang: Beckman Institute, University of Illinois at Urbana-Champaign, USA. huang@ifp.uiuc.edu

This work has been partially funded by NSF Grant IIS 00-85980. 


\begin{abstract}
Automatic classification is one of the basic tasks required in any pattern recognition and human computer interaction application. In this paper we discuss training probabilistic classifiers with labeled and unlabeled data. We provide a new analysis that shows under what conditions unlabeled data can be used in learning to improve classification performance. We also show that if the conditions are violated, using unlabeled data can be detrimental to classification performance. We discuss the implications of this analysis to a specific type of probabilistic classifiers, Bayesian networks, and propose a new structure learning algorithm that can utilize unlabeled data to improve classification. Finally, we show how the resulting algorithms are successfully employed in two applications related to human-computer interaction and pattern recognition; facial expression recognition and face detection.
\end{abstract}

\title{
Index Terms
}

Semi-supervised learning, generative models, facial expression recognition, face detection, unlabeled data, Bayesian network classifiers.

\section{INTRODUCTION}

Many pattern recognition and human computer interaction applications require the design of classifiers. Classifiers are either designed from expert knowledge or from training data. Training data can be either labeled or unlabeled. In many applications, obtaining fully labeled training sets is a difficult task; labeling is usually done using human expertise, which is expensive, time consuming and error prone. Obtaining unlabeled data is usually easier since it involves collecting data that are known to belong to one of the classes without having to label it. For example, in facial expression recognition, it is easy to collect videos of people displaying expressions, but it is very tedious and difficult to label the video to the corresponding expressions. Learning with both labeled and unlabeled data is known as semi-supervised learning.

We start with a general analysis of semi-supervised learning for probabilistic classifiers. The goal of the analysis is to show under what conditions unlabeled data can be used to improve the classification performance. We review maximum likelihood estimation when learning with labeled and unlabeled data. We provide an asymptotic analysis of the value of unlabeled data to show that unlabeled data help in reducing the estimator's variance. We show that when the assumed probabilistic model matches the data generating distribution, the reduction in variance leads to an improved classification accuracy; a situation that has been analyzed before $[1,2]$. 
However, we show that when the assumed probabilistic model does not match the true data generating distribution, using unlabeled data can be detrimental to the classification accuracy; a phenomenon that has been generally ignored or misinterpreted by previous researchers who observed it empirically before [1,3,4]. This new result emphasizes the importance of using correct modeling assumption when learning with unlabeled data.

We also present in this paper an analysis of semi-supervised learning for classifiers based on Bayesian networks. While in many classification problems simple structures learned with just labeled data have been used successfully (e.g., the Naive-Bayes classifier [5,6]), such structures fail when trained with both labeled and unlabeled data [7]. Bayesian networks are probabilistic classifiers, in which the joint distribution of the features and class variables is specified using a graphical model [8]. The graphical representation has several advantages. Among them are the existence of algorithms for inferring the class label, the ability to intuitively represent fusion of different modalities with the graph structure $[9,10]$, the ability to perform classification and learning without complete data, and most importantly, the ability to learn with both labeled and unlabeled data. We discuss possible strategies for choosing a good graphical structure and argue that in many problems, it is necessary to search for such a structure. Most structure search algorithms are driven by likelihood based cost functions, which are potentially inadequate for classification $[11,12]$ due to their attempt to maximize the overall likelihood of the data, while largely ignoring the important quantity for classification; the class a-posteriori likelihood. As such, we propose a classification driven stochastic structure search algorithm (SSS), which combines both labeled and unlabeled data to train the classifier and to search for a better performing Bayesian network structure.

Following the new understanding of the limitations imposed by the properties of unlabeled data, and equipped with an algorithm to overcome these limitations, we apply the Bayesian network classifiers to two human-computer interaction problems: facial expression recognition and face detection. In both of these applications, obtaining unlabeled training data is relatively easy. However, in both cases, labeling of the data is difficult. For facial expression recognition, accurate labeling requires expert knowledge [13] and for both applications, labeling of a large amount of data is time consuming for the human labeler. We show that Bayesian network classifiers trained with structure search benefit from semi-supervised learning in both of these problems. 
The remainder of the paper is organized as follows. In Section II we discuss the value of unlabeled data and illustrate the possibility of unlabeled data to degrade the classification performance. In Section III we propose possible solutions for Bayesian network classifiers to benefit from unlabeled data by learning the network structure. We introduce a new stochastic structure search algorithm and empirically show its ability to learn with both labeled and unlabeled data using datasets from the UCI machine learning repository [14]. In Section IVA we describe the components of our real-time face recognition system, including the real-time face tracking system and the features extracted for classification of facial expressions. We perform experiments of our facial expression recognition system using two databases and show the ability to utilize unlabeled data to enhance the classification performance, even with a small labeled training set. Experiments of Bayesian network classifiers for face detection are given in Section IV-B. We have concluding remarks in Section V.

\section{LEARNING A CLASSIFIER FROM LABELED AND UNLABELED TRAINING DATA}

The goal is to classify an incoming vector of observables $\mathbf{X}$. Each instantiation of $\mathbf{X}$ is a sample. There exists a class variable $C$; the values of $C$ are the classes. We want to build classifiers that receive a sample $\mathrm{x}$ and output a class. We assume $0-1$ loss, and consequently our objective is to minimize the probability of error (classification error). If we knew exactly the joint distribution $p(C, \mathbf{X})$, the optimal rule would be to choose the class value with the maximum a-posteriori probability, $p(C \mid \mathbf{x})$ [15]. This classification rule attains the minimum possible classification error, called the Bayes error.

We take that the probabilities of $(C, \mathbf{X})$, or functions of these probabilities, are estimated from data and then "plugged" into the optimal classification rule. We assume that a parametric model $p(C, \mathbf{X} \mid \theta)$ is adopted. An estimate of $\theta$ is denoted by $\hat{\theta}$ and we denote throughout by $\hat{\theta}^{*}$ the asymptotic value of $\hat{\theta}$. If the distribution $p(C, \mathbf{X})$ belongs to the family $p(C, \mathbf{X} \mid \theta)$, we say the "model is correct"; otherwise we say the "model is incorrect". We use "estimation bias" loosely to mean the expected difference between $p(C, \mathbf{X})$ and the estimated $p(C, \mathbf{X} \mid \hat{\theta})$.

We consider the following scenario. A sample $(c, \mathbf{x})$ is generated from $p(C, \mathbf{X})$. The value $c$ is then either revealed, and the sample is a labeled one; or the value $c$ is hidden, and the sample is an unlabeled one. The probability that any sample is labeled, denoted by $\lambda$, is fixed, known, 
and independent of the samples ${ }^{1}$. Thus, the same underlying distribution $p(C, \mathbf{X})$ generates both labeled and unlabeled data. It is worth noting that we assume the revealed label is correct and is not corrupted by noise; the case of noisy labels has been studied in various works (such as [17-20], chapter 2 of [21]). Extending our analysis to the noisy labeled case is beyond the scope of this paper.

Given a set of $N_{l}$ labeled samples and $N_{u}$ unlabeled samples, we use maximum likelihood for estimating $\hat{\theta}$. We consider distributions that decompose $p(C, \mathbf{X} \mid \theta)$ as $p(\mathbf{X} \mid C, \theta) p(C \mid \theta)$, where both $p(\mathbf{X} \mid C, \theta)$ and $p(C \mid \theta)$ depend explicitly on $\theta$. This is known as a generative model. The log-likelihood function of a generative model for a dataset with labeled and unlabeled data is:

$$
L(\theta)=L_{l}(\theta)+L_{u}(\theta)+\log \left(\lambda^{N_{l}}(1-\lambda)^{N_{u}}\right),
$$

where $L_{u}(\theta)=\sum_{j=\left(N_{l}+1\right)}^{N_{l}+N_{u}} \log \left[p\left(\mathbf{x}_{j} \mid \theta\right)\right]$, and $L_{l}(\theta)=\sum_{i=1}^{N_{l}} \log \left[\prod_{C}\left(p\left(C=c^{\prime} \mid \theta\right) p\left(\mathbf{x}_{i} \mid c^{\prime}, \theta\right)\right)^{I_{\left\{C=c^{\prime}\right\}}\left(c_{i}\right)}\right]$ with $I_{A}(Z)$ the indicator function: 1 if $Z \in A$; 0 otherwise. $L_{l}(\theta)$ and $L_{u}(\theta)$ are the likelihoods of the labeled and unlabeled data, respectively.

Statistical intuition suggests that it is reasonable to expect an average improvement in classification performance for any increase in the number of samples (labeled or unlabeled). Indeed, the existing literature presents several empirical and theoretical findings that do indicate positive value in unlabeled data. Cooper and Freeman [22] were optimistic enough about unlabeled data so as to title their work as "On the asymptotic improvement in the outcome of supervised learning provided by additional nonsupervised learning" . Other early studies, such as [23-25] further strengthened the assertion that unlabeled data should be used whenever available. Castelli [26] and Ratsaby and Venkatesh [27] showed that unlabeled data are always asymptotically useful for classification. Krishnan and Nandy [19,20] extended the results of [25] to provide efficiency results for discriminant and logistic-normal models for samples that are labeled stochastically. It should be noted that such previous theoretical work makes the critical assumption that $p(C, \mathbf{X})$ belongs to the family of models $p(C, \mathbf{X} \mid \theta)$ (that is, the "model is correct").

There has also been recent applied work on semi-supervised learning [1,3-5, 28-32]. Overall, these publications advance an optimistic view of the labeled-unlabeled data problem, where unlabeled data can be profitably used whenever available.

\footnotetext{
${ }^{1}$ This is different from [3] and [16], where $\lambda$ is a parameter that can be set.
} 


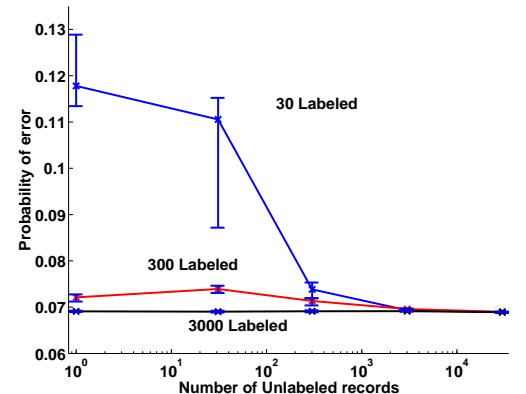

(a)

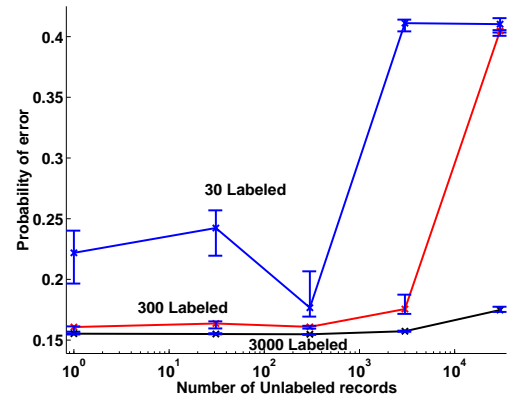

(b)

Fig. 1. Naive Bayes classifier from data generated from a Naive Bayes model (a) and a TAN model (b). Each point summarizes 10 runs of each classifier on testing data; bars cover 30 to 70 percentiles.

However, a more detailed analysis of current applied results does reveal some puzzling aspects of unlabeled data. Researchers have reported cases where the addition of unlabeled data degraded the performance of the classifiers when compared to the case in which unlabeled data is not used. These cases were not specific to one type of data, but for different kinds, such as sensory data [1], computer vision [5], and text classification [3,4].

To explain the phenomenon, we began by performing extensive experiments providing empirical evidence that degradation of performance is directly related to incorrect modeling assumptions [33-35]. Consider Figure 1, which shows two typical results. Here we estimated the parameters of a Naive Bayes classifier with 10 features using the Expectation-Maximization (EM) algorithm [36] with varying numbers of labeled and unlabeled data. Figure 1 shows classification performance when the underlying model actually has a Naive Bayes structure (left), and when the underlying model is not Naive Bayes (right). The result is clear: when we estimate a Naive Bayes classifier with data generated from a Naive Bayes model, more unlabeled data help; when we estimate a Naive Bayes classifier with data that do not come from a corresponding model, more unlabeled data can degrade performance (even for the case of 30 labeled and 30000 unlabeled samples!).

To provide a theoretical explanation to the empirical evidence, we derived the asymptotic properties of maximum likelihood estimators for the labeled-unlabeled case. The analysis, presented in the remainder of this section, provides a unified explanation of the behavior of classifiers for both cases; when the model is correct and when it is not. 


\section{A. The value of unlabeled data in maximum likelihood estimation}

We base our analysis on the work of White [37] on the properties of maximum likelihood estimators - properties that hold for the case of model correctness and model incorrectness. In [37], Theorems 3.1, 3.2, and 3.3 showed that under suitable regularity conditions ${ }^{2}$, maximum likelihood estimators converge to a parameter set $\theta^{*}$ that minimizes the Kullback-Liebler (KL) distance between the assumed family of distributions, $p(Y \mid \theta)$, and the true distribution, $p(Y)$. White [37] also shows that the estimator is asymptotically Normal, i.e., $\sqrt{N}\left(\hat{\theta}_{N}-\theta^{*}\right) \sim$ $\mathcal{N}\left(0, C_{Y}(\theta)\right)$ as $N$ (the number of samples) goes to infinity. $C_{Y}(\theta)$ is a covariance matrix equal to $A_{Y}(\theta)^{-1} B_{Y}(\theta) A_{Y}(\theta)^{-1}$, evaluated at $\theta^{*}$, where $A_{Y}(\theta)$ and $B_{Y}(\theta)$ are matrices whose $(i, j)$ 'th element $(i, j=1, \ldots, d$, where $d$ is the number of parameters) is given by:

$$
\begin{aligned}
& A_{Y}(\theta)=E\left[\partial^{2} \log p(Y \mid \theta) / \partial \theta_{i} \theta_{j}\right], \\
& B_{Y}(\theta)=E\left[\left(\partial \log p(Y \mid \theta) / \partial \theta_{i}\right)\left(\partial \log p(Y \mid \theta) / \partial \theta_{j}\right)\right] .
\end{aligned}
$$

Using these definitions and general result, we obtain:

Theorem 1: Consider supervised learning where samples are randomly labeled with probability $\lambda$. Assuming identifiability for the marginal distributions of $\mathbf{X}$, then the value of $\theta^{*}$, the limiting value of maximum likelihood estimates, is:

$$
\arg \max _{\theta}(\lambda E[\log p(C, \mathbf{X} \mid \theta)]+(1-\lambda) E[\log p(\mathbf{X} \mid \theta)])
$$

where the expectations are with respect to $p(C, \mathbf{X})$. Additionally, $\sqrt{N}\left(\hat{\theta}_{N}-\theta^{*}\right) \sim \mathcal{N}\left(0, C_{\lambda}(\theta)\right)$ as $N \rightarrow \infty$, where $C_{\lambda}(\theta)$ is given by:

$$
\begin{aligned}
& C_{\lambda}(\theta)=A_{\lambda}(\theta)^{-1} B_{\lambda}(\theta) A_{\lambda}(\theta)^{-1} \text { with, } \\
& A_{\lambda}(\theta)=\left(\lambda A_{(C, \mathbf{X})}(\theta)+(1-\lambda) A_{\mathbf{X}}(\theta)\right) \text { and } \\
& B_{\lambda}(\theta)=\left(\lambda B_{(C, \mathbf{X})}(\theta)+(1-\lambda) B_{\mathbf{X}}(\theta)\right),
\end{aligned}
$$

evaluated at $\theta^{*}$, where $A_{\mathbf{X}}(\theta), A_{(C, \mathbf{X})}(\theta), B_{\mathbf{X}}(\theta)$ and $B_{(C, \mathbf{X})}(\theta)$ are the $A$ and $B$ defined in Expression 2, with $Y$ replaced by $(C, \mathbf{X})$ or $\mathbf{X}$.

Proof. Denote by $\tilde{C}$ a random variable that assumes the same values of $C$ plus the "unlabeled" value $u$. We have $p(\tilde{C} \neq u)=\lambda$. The observed samples are realizations of $(\tilde{C}, \mathbf{X})$, so we can

\footnotetext{
${ }^{2}$ The conditions ensure the existence of the derivatives defined below and the expectations used in Theorem 1 .
} 
write the probability distribution of a sample compactly as follows:

$$
\tilde{p}(\tilde{C}=c, \mathbf{X}=\mathbf{x})=(\lambda p(C=c, \mathbf{X}=\mathbf{x}))^{I_{\{\tilde{C} \neq u\}}(c)}((1-\lambda) p(\mathbf{X}=\mathbf{x}))^{I_{\{\tilde{C}=u\}}(c)}
$$

where $p(\mathbf{X})$ is a mixture density obtained from $p(C, \mathbf{X})$. Accordingly, the parametric model adopted for $(\tilde{C}, \mathbf{X})$ is:

$$
\tilde{p}(\tilde{C}=c, \mathbf{X}=\mathbf{x} \mid \theta)=(\lambda p(C=c, \mathbf{X}=\mathbf{x} \mid \theta))^{I_{\{\tilde{C} \neq u\}}(c)}((1-\lambda) p(\mathbf{X}=\mathbf{x} \mid \theta))^{I_{\{\tilde{C}=u\}}(c)} .
$$

From White's results stated above, we know that $\theta^{*}$ maximizes $E[\log \tilde{p}(\tilde{C}, \mathbf{X} \mid \theta)]$ (expectation with respect to $\tilde{p}(\tilde{C}, \mathbf{X})$ ). We have:

$$
\begin{aligned}
E[\log \tilde{p}(\tilde{C}, \mathbf{X} \mid \theta)]= & E\left[I_{\{\tilde{C} \neq u\}}(\tilde{C})(\log \lambda+\log p(C, \mathbf{X} \mid \theta))+I_{\{\tilde{C}=u\}}(\tilde{C})(\log (1-\lambda)+\log p(\mathbf{X} \mid \theta))\right] \\
= & \lambda \log \lambda+(1-\lambda) \log (1-\lambda)+ \\
& E\left[I_{\{\tilde{C} \neq u\}}(\tilde{C}) \log p(C, \mathbf{X} \mid \theta)\right]+E\left[I_{\{\tilde{C}=u\}}(\tilde{C}) \log p(\mathbf{X} \mid \theta)\right] .
\end{aligned}
$$

The first two terms of this expression are irrelevant to maximization with respect to $\theta$. The last two terms are equal to

$$
\lambda E[\log p(C, \mathbf{X} \mid \theta) \mid \tilde{C} \neq u]+(1-\lambda) E[\log p(\mathbf{X} \mid \theta) \mid \tilde{C}=u] .
$$

As we have $\tilde{p}(\tilde{C}, \mathbf{X} \mid \tilde{C} \neq u)=p(C, \mathbf{X})$ and $\tilde{p}(\mathbf{X} \mid \tilde{C}=u)=p(\mathbf{X})$ (Expression (5)), the last expression is equal to

$$
\lambda E[\log p(C, \mathbf{X} \mid \theta)]+(1-\lambda) E[\log p(\mathbf{X} \mid \theta)]
$$

where the last two expectations are now with respect to $p(C, \mathbf{X})$. Thus we obtain Expression (3). Expression (4) follows directly from White's theorem and Expression (3), replacing $Y$ by $(C, \mathbf{X})$ and $\mathrm{X}$ where appropriate.

A few observations can be made from the theorem. First, Expression (3) indicates that semisupervised learning can be viewed asymptotically as a "convex" combination of supervised and unsupervised learning. The objective function for semi-supervised learning is a combination of the objective function for supervised learning $(E[\log p(C, \mathbf{X} \mid \theta)])$ and the objective function for unsupervised learning $(E[\log p(\mathbf{X} \mid \theta)])$. Second, because the asymptotic covariance matrix is positive definite as $B_{Y}(\theta)$ is positive definite and $A_{Y}(\theta)$ is symmetric for any $Y$, $\theta A(\theta)^{-1} B_{Y}(\theta) A(\theta)^{-1} \theta^{T}=w(\theta) B_{Y}(\theta) w(\theta)^{T}>0$, where $w(\theta)=\theta A_{Y}(\theta)^{-1}$. We see that 
asymptotically, an increase in $N$, the number of labeled and unlabeled samples, will lead to a reduction in the variance of $\hat{\theta}$. Such a guarantee can perhaps be the basis for the optimistic view that unlabeled data should always be used to improve classification accuracy. In the following, we show this view is valid when the model is correct, and that it is not always valid when the model is incorrect.

\section{B. Model is correct}

Suppose first that the family of distributions $P(C, \mathbf{X} \mid \theta)$ contains the distribution $P(C, \mathbf{X})$; that is, $P\left(C, \mathbf{X} \mid \theta_{\top}\right)=P(C, \mathbf{X})$ for some $\theta_{\top}$. Under this condition, the maximum likelihood estimator is consistent, thus, $\theta_{\lambda=1}^{*}=\theta_{\lambda=0}^{*}=\theta_{\top}$ given identifiability. Thus, $\theta_{\lambda}^{*}=\theta_{\top}$ for any $0 \leq \lambda \leq 1$

Shahshahani and Landgrebe [1] suggested using the Taylor expansion of the classification error around $\theta_{\top}$ to link the decrease in variance associated with unlabeled data to a decrease in classification error. They show that the smaller the variance of the estimator, the smaller the classification error, and since the variance of the estimator is smaller as the number of samples increases (labeled or unlabeled), adding the unlabeled data would reduce classification error. A more formal, but less general, argument is presented by Ganesalingam and McLachlan [25] as they compare the relative efficiency of labeled and unlabeled data. Castelli [26] also derives a Taylor expansion of the classification error, to study estimation of the mixing factors, $p(C=c)$; the derivation is very precise and states all the required assumptions.

\section{Model is incorrect}

We now study the more realistic scenario where the distribution $P(C, \mathbf{X})$ does not belong to the family of distributions $P(C, \mathbf{X} \mid \theta)$. In view of Theorem 1 , it is perhaps not surprising that unlabeled data can have the deleterious effect observed occasionally in the literature. Suppose that $\theta_{u}^{*} \neq \theta_{l}^{*}$ and that $\mathbf{e}\left(\theta_{u}^{*}\right)>\mathbf{e}\left(\theta_{l}^{*}\right)$, as in the example in the next section, where $\theta_{l}^{*}=\theta_{\lambda=1}^{*}$ and $\theta_{u}^{*}=\theta_{\lambda=0}^{*} \cdot{ }^{3}$ If we observe a large number of labeled samples, the classification error is

\footnotetext{
${ }^{3} \mathrm{We}$ have to handle a difficulty with $\mathbf{e}\left(\theta_{u}^{*}\right)$ : given only unlabeled data, there is no information to decide the labels for decision regions, and then the classification error is $1 / 2$ [26]. Instead of defining $\mathbf{e}\left(\theta_{u}^{*}\right)$ as the error for $\lambda=0$, we could define $\mathbf{e}\left(\theta_{u}^{*}\right)$ as the error of $\lambda$ approaching 0 .
} 
approximately $\mathbf{e}\left(\theta_{l}^{*}\right)$. If we then collect more samples, most of which unlabeled, we eventually reach a point where the classification error approaches $\mathbf{e}\left(\theta_{u}^{*}\right)$. So, the net result is that we started with classification error close to $\mathbf{e}\left(\theta_{l}^{*}\right)$, and by adding a large number of unlabeled samples, classification performance degraded. The basic fact here is that estimation and classification bias are affected differently by different values of $\lambda$. Hence, a necessary condition for this kind of performance degradation is that $\mathbf{e}\left(\theta_{u}^{*}\right) \neq \mathbf{e}\left(\theta_{l}^{*}\right)$; a sufficient condition is that $\mathbf{e}\left(\theta_{u}^{*}\right)>\mathbf{e}\left(\theta_{l}^{*}\right)$.

1) Example: Bivariate Gaussians with spurious correlation: The previous discussion alluded to the possibility that $\mathbf{e}\left(\theta_{u}^{*}\right)>\mathbf{e}\left(\theta_{l}^{*}\right)$ when the model is incorrect. To the skeptical reader who still may think that this will not occur in practice, or that numerical algorithms, such as EM, are the cause of performance degradation, we analytically show how this occurs with an example of obvious practical significance. More examples are provided in [38] and [34].

We will assume that bivariate Gaussian samples $(X, Y)$ are observed. The only modeling error is an ignored dependency between observables. This type of modeling error is quite common in practice and has been studied in the context of supervised learning $[39,40]$. Is it often argued that ignoring some dependencies can be a positive decision, as we may see a reduction in the number of parameters to be estimated and a reduction on the variance of estimates [41].

Example 1: Consider real-valued observations $(X, Y)$ taken from two classes $c^{\prime}$ and $c^{\prime \prime}$. We know that $X$ and $Y$ are Gaussian variables, and we know their means and variances given the class $C$. The mean of $(X, Y)$ is $(0,3 / 2)$ conditional on $\left\{C=c^{\prime}\right\}$, and $(3 / 2,0)$ conditional on $\left\{C=c^{\prime \prime}\right\}$. Variances for $X$ and for $Y$ conditional on $C$ are equal to 1 . We do not know, and have to estimate, the mixing factor $\eta=p\left(C=c^{\prime}\right)$. The data is sampled from a distribution with mixing factor equal to $3 / 5$.

We want to obtain a Naive-Bayes classifier that can approximate $p(C \mid X, Y)$; Naive-Bayes classifiers are based on the assumption that $X$ and $Y$ are independent given $C$. Suppose that $X$ and $Y$ are independent conditional on $\left\{C=c^{\prime}\right\}$ but that $X$ and $Y$ are dependent conditional on $\left\{C=c^{\prime \prime}\right\}$. This dependency is manifested by a correlation

$$
\rho=E\left[\left(X-E\left[X \mid C=c^{\prime \prime}\right]\right)\left(Y-E\left[Y \mid C=c^{\prime \prime}\right]\right) \mid C=c^{\prime \prime}\right]=4 / 5
$$

. If we knew the value of $\rho$, we would obtain an optimal classification boundary on the plane $X \times Y$. This optimal classification boundary is shown in Figure 2, and is defined by the function

$$
y=\left(40 x-87+\sqrt{5265-2160 x+576 x^{2}+576 \log (100 / 81)}\right) / 32 .
$$


Under the incorrect assumption that $\rho=0$, the classification boundary is then linear:

$$
y=x+2 \log ((1-\hat{\eta}) / \hat{\eta}) / 3,
$$

and consequently it is a decreasing function of $\hat{\eta}$. With labeled data we can easily obtain $\hat{\eta}$ (a sequence of Bernoulli trials); then $\eta_{l}^{*}=3 / 5$ and the classification boundary is given by $y=x-0.27031$.

Note that the (linear) boundary obtained with labeled data is not the best possible linear boundary. We can in fact find the best possible linear boundary of the form $y=x+\gamma$. For any $\gamma$, the classification error $\mathbf{e}(\gamma)$ is

$$
\frac{3}{5} \int_{-\infty}^{\infty} \int_{-\infty}^{x+\gamma} N\left(\left[\begin{array}{c}
0 \\
3 / 2
\end{array}\right], \operatorname{diag}[1,1]\right) d y d x+\frac{2}{5} \int_{-\infty}^{\infty} \int_{x+\gamma}^{\infty} N\left(\left[\begin{array}{c}
3 / 2 \\
0
\end{array}\right],\left[\begin{array}{cc}
1 & 4 / 5 \\
4 / 5 & 1
\end{array}\right]\right) d y d x .
$$

By interchanging differentiation with respect to $\gamma$ with integration, it is possible to obtain $d \mathbf{e}(\gamma) / d \gamma$ in closed form. The second derivative $d^{2} \mathbf{e}(\gamma) / d \gamma^{2}$ is positive when $\gamma \in[-3 / 2,3 / 2]$; consequently there is a single minimum that can be found by solving $d \mathbf{e}(\gamma) / d \gamma=0$. We find the minimizing $\gamma$ to be $(-9+2 \sqrt{45 / 4+\log (400 / 81)}) / 4 \approx-0.45786$. The line $y=x-0.45786$ is the best linear boundary for this problem. If we consider the set of lines of the form $y=x+\gamma$, we see that the farther we go from the best line, the larger the classification error. Figure 2 shows the linear boundary obtained with labeled data and the best possible linear boundary. The boundary from labeled data is "above" the best linear boundary.

Now consider the computation of $\eta_{u}^{*}$. Using Theorem 1 the asymptotic estimate with unlabeled data is:

$$
\begin{aligned}
\eta_{u}^{*}=\arg \max _{\eta \in[0,1]} \int_{-\infty}^{\infty} \int_{-\infty}^{\infty} \log \left(\eta N\left([0,3 / 2]^{T}, \operatorname{diag}[1,1]\right)+(1-\eta) N\left([3 / 2,0]^{T}, \operatorname{diag}[1,1]\right)\right) \\
\\
\left((3 / 5) N\left([0,3 / 2]^{T}, \operatorname{diag}[1,1]\right)+(2 / 5) N\left(\left[\begin{array}{c}
3 / 2 \\
0
\end{array}\right],\left[\begin{array}{cc}
1 & 4 / 5 \\
4 / 5 & 1
\end{array}\right]\right)\right) d y d x .
\end{aligned}
$$

The second derivative of this double integral is always negative (as can be seen interchanging differentiation with integration), so the function is concave and there is a single maximum. We can search for the zero of the derivative of the double integral with respect to $\eta$. We obtain this value numerically, $\eta_{u}^{*} \approx 0.54495$. Using this estimate, the linear boundary from unlabeled data is $y=x-0.12019$. This line is "above" the linear boundary from labeled data, and, given the previous discussion, leads to a larger classification error than the boundary from labeled data. 


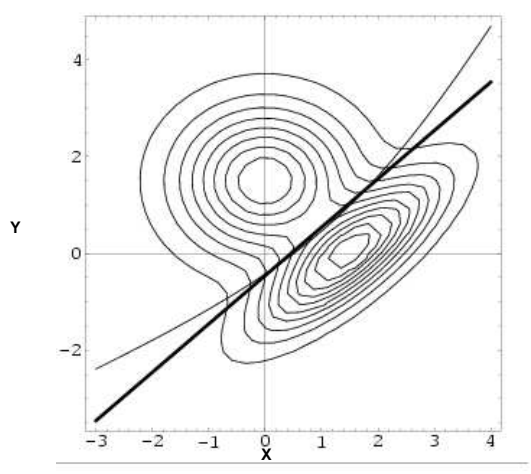

(a)

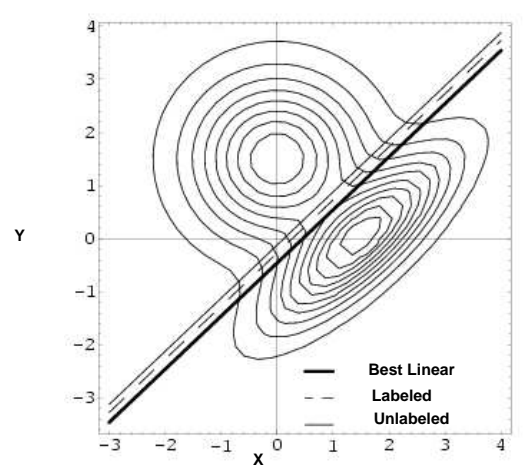

(b)

Fig. 2. Graphs for Example 1. (a) contour plots of the mixture $p(X, Y)$, the optimal classification boundary (quadratic curve) and the best possible classification boundary of the form $y=x+\gamma$. (b) the same contour plots, and the best linear boundary (lower line), the linear boundary obtained from labeled data (middle line) and the linear boundary obtained from unlabeled data (upper line); thus the classification error of the classifier obtained with unlabeled data is larger than that of the classifier obtained with labeled data.

We have: $\mathbf{e}(\gamma)=0.06975 ; \mathbf{e}\left(\theta_{l}^{*}\right)=0.07356 ; \mathbf{e}\left(\theta_{u}^{*}\right)=0.08141$. The boundary obtained from unlabeled data is also shown in Figure 2.

This example suggests the following situation. Suppose we collect a large number $N_{l}$ of labeled samples from $p(C, X)$, with $\eta=3 / 5$ and $\rho=4 / 5$. The labeled estimates form a sequence of Bernoulli trials with probability $3 / 5$, so the estimates quickly approach $\eta_{l}^{*}$ (the variance of $\hat{\eta}$ decreases as $6 /\left(25 N_{l}\right)$ ). If we add a very large amount of unlabeled data to our data, $\hat{\eta}$ approaches $\eta_{u}^{*}$ and the classification error increases.

\section{Finite sample effects}

The asymptotic analysis of semi-supervised learning suffices to show the fundamental problem that can occur when learning with unlabeled data. The focus on asymptotics is adequate as we want to eliminate phenomena that can vary from dataset to dataset. If $\mathbf{e}\left(\theta_{l}^{*}\right)$ is smaller than $\mathbf{e}\left(\theta_{u}^{*}\right)$, then a large enough labeled dataset can be dwarfed by a much larger unlabeled dataset - the classification error using the whole dataset can be larger than the classification error using the labeled data only. But what occurs with finite sample size datasets? We performed extensive experiments with real and artificial datasets of various sizes [7,34]. Throughout our experiments we used the EM algorithm to maximize the likelihood (Expression (1)), and we started the 


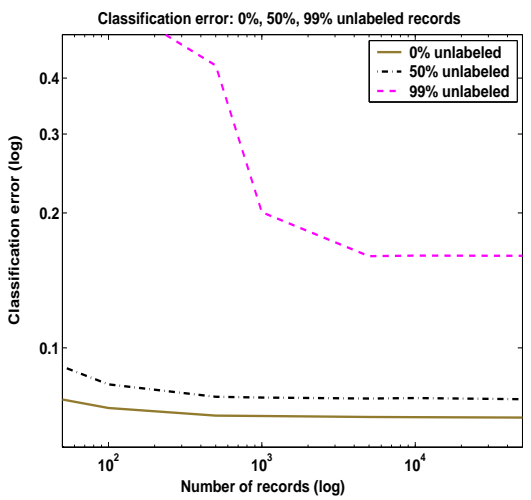

(a)

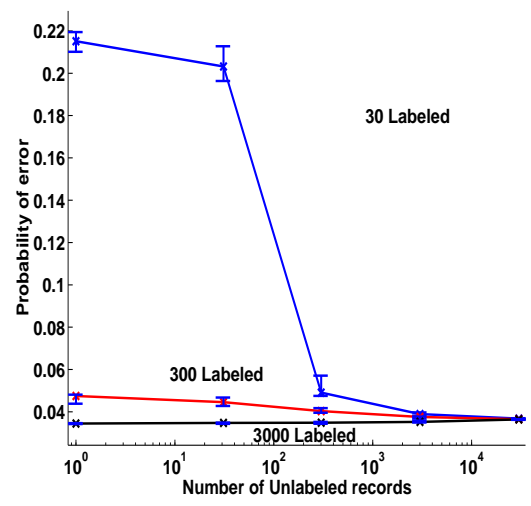

(b)

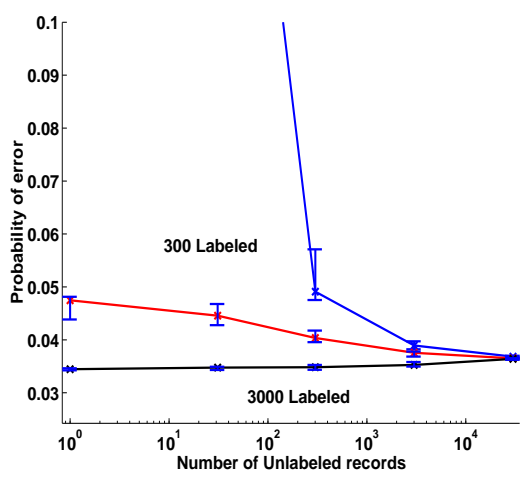

(c)

Fig. 3. (a) LU-graphs for the example with two Gaussian observables. Each sample in each graph is the average of 100 trials; classification error was obtained by testing in 10000 labeled samples drawn from the correct model. (b) Naive Bayes classifiers from data generated from a TAN model (introduced in Section III) with 49 observables (each variable with 2 to 4 values); points in the graphs summarize 10 runs on testing data (bars cover 30 to 70 percentiles). (c) Same graph as (b), enlarged. Note that unlabeled data does lead to a significant improvement in performance when added to 30 or 300 labeled samples. There is performance degradation in the presence of 3000 labeled samples.

EM algorithm with the parameters obtained using labeled data, as these starting points can be obtained in closed-form.

To visualize the effect of labeled and unlabeled samples, we suggest that the most profitable strategy is to fix the percentage of unlabeled samples $(\lambda)$ among all training samples. We then plot classification error against the number of training samples. Call such a graph a LU-graph.

Example 2: Consider a situation where we have a binary class variable $C$ with values $c^{\prime}$ and $c^{\prime \prime}$, and $p\left(C=c^{\prime}\right)=0.4017$. We also have two real-valued observables $X$ and $Y$ with distributions:

$$
\begin{gathered}
p\left(X \mid c^{\prime}\right)=N(2,1), \quad p\left(X \mid c^{\prime \prime}\right)=N(3,1), \\
p\left(Y \mid c^{\prime}, x\right)=N(2,1), \quad p\left(Y \mid c^{\prime \prime}, x\right)=N(1+2 x, 1) .
\end{gathered}
$$

There is dependency between $Y$ and $X$ conditional on $\left\{C=c^{\prime \prime}\right\}$. Suppose we build a Naive Bayes classifier for this problem. Figure 3(a) shows the LU-graphs for 0\% unlabeled samples, 50\% unlabeled samples and 99\% unlabeled samples, averaging over a large ensemble of classifiers. The asymptotes converge to different values. Suppose then that we started with 50 labeled samples as our training data. Our classification error would be about $7.8 \%$, as we can 
see in the LU-graph for $0 \%$ unlabeled data. Suppose we added 50 labeled samples; we would obtain a classification error of about $7.2 \%$. Now suppose we added 100 unlabeled samples. We would move from the $0 \%$ LU-graph to the $50 \%$ LU-graph. Classification error would increase to $8.2 \%$ ! And if we then added 9800 unlabeled samples, we would move to the $99 \%$ LU-graph, with classification error about $16.5 \%$ - more than twice the error we had with just 50 labeled samples.

It should be noted that in difficult classification problems, where LU-graphs decrease very slowly, unlabeled data may improve classification performance for certain regions of the LU graphs. Problems with a large number of observables and parameters should require more training data, so we can expect that such problems benefit more consistently from unlabeled data. Figures 3(b-c) illustrate this possibility for a Naive-Bayes classifier with 49 features. Another possible phenomenon is that the addition of a substantial number of unlabeled samples may reduce variance and decrease classification error, but an additional, much larger, pool of unlabeled data can eventually add enough bias so as to increase classification error. Such a situation is likely to have happened in some of the results reported by Nigam et al [3], where classification errors go up and down as more unlabeled samples are added.

In summary, semi-supervised learning displays an odd failure of robustness: for certain modeling errors, more unlabeled data can degrade classification performance. Estimation bias is the central factor in this phenomenon, as the level of bias depends on the ratio of labeled to unlabeled samples. Most existing theoretical results on semi-supervised learning are based on the assumption of no modeling error, and consequently bias has not been an issue so far.

\section{SEMI-SUPERVISED LEARNING FOR BAYESIAN NETWORK CLASSIFIERS}

We now turn our attention to the implication of the previous analysis to Bayesian network classifiers. As stated before, we chose Bayesian network classifiers for several reasons; classification is possible with missing data in general and unlabeled data in particular, the graphical representation is intuitive and can be easily expanded to add different features and modalities, and there are efficient algorithms for inference.

A Bayesian network [8] is composed of a directed acyclic graph in which every node is associated with a variable $X_{i}$ and with a conditional distribution $p\left(X_{i} \mid \Pi_{i}\right)$, where $\Pi_{i}$ denotes the parents of $X_{i}$ in the graph. The joint probability distribution is factored to the collection of 
conditional probability distributions of each node in the graph as:

$$
p\left(X_{1}, \ldots, X_{n}\right)=\prod_{i=1}^{n} p\left(X_{i} \mid \Pi_{i}\right) .
$$

The directed acyclic graph is the structure, and the distributions $p\left(X_{i} \mid \Pi_{i}\right)$ represent the parameters of the network. Consider now that data generated by a distribution $p(C, \mathbf{X})$ are collected. We say that the assumed structure for a network, $S^{\prime}$, is correct when it is possible to find a distribution, $p\left(C, \mathbf{X} \mid S^{\prime}\right)$, that matches the data generating distribution $p(C, \mathbf{X})$; otherwise, the structure is incorrect ${ }^{4,5}$. Maximum likelihood estimation is one of the main methods to learn the parameters of the network. When there are missing data in training set, the EM algorithm can be used to maximize the likelihood.

As a direct consequence of the analysis in the previous section, a Bayesian network that has the correct structure and the correct parameters is also optimal for classification because the a-posteriori distribution of the class variable is accurately represented. Thus, there is great motivation for obtaining the correct structure when conducting semi-supervised learning. Somewhat surprisingly, the option of searching for better structures has not been proposed by researchers who have previously witnessed the performance degradation when learning with unlabeled data. In the following sections, we describe different strategies for learning Bayesian network classifiers with labeled and unlabeled data.

\section{A. Switching between simple models and structure learning}

If we observe performance degradation, we may try to find the "correct" structure for our Bayesian network classifier. Alas, learning Bayesian network structure is not a trivial task.

One attempt, perhaps the simplest, to overcome performance degradation from unlabeled data could be to assume a very simple model (such as the Naive Bayes), which is typically not the correct structure, and switch to a more complex model as soon as degradation is detected. One such family of models is the Tree-Augmented Naive-Bayes (TAN) [11]. While such a strategy has no guarantees to find the correct structure, the existence of an efficient algorithm for learning

\footnotetext{
${ }^{4}$ These definitions follow directly from the definitions of correct and incorrect models described in the previous section.

${ }^{5}$ There is not necessarily a unique correct structure, e.g., if a structure is correct (as defined above), all structures that are from the same Markov equivalent class are also correct since causality is not an issue.
} 
the TAN models, both in the supervised case [11] and in the semi-supervised case [7,42], makes switching to TAN models attractive. However, while both the Naive-Bayes and TAN classifiers have been observed to be successful in the supervised case [41], the same success is not always observed for the semi-supervised case (Section III-C).

When such simple strategies fail, performing unconstrained structure learning is the alternative. There are various approaches for learning the structure of Bayesian networks, using different criteria in an attempt to find the correct structure.

The first class of structure learning methods we consider is the class of independence-based methods, also known as constraint-based or test-based methods. There are several such algorithms [43-45], all of them can obtain the correct structure if there are fully reliable independence tests available; however not all of them are appropriate for classification. The Cheng-Bell-Liu algorithms (CBL1 and CBL2) seem particularly well-suited for classification, as they strive to keep the number of edges in the Bayesian networks as small as possible and the performance of CBL1 on labeled data only has been reported to surpass the performance of TAN [46]. Because independence-based algorithms like CBL1 do not explicitly optimize a metric, they cannot handle unlabeled data directly through an optimization scheme like EM. To handle unlabeled data, the following strategy was derived (denoted as EM-CBL): Start by learning a Bayesian network with the available labeled data; then use EM to process unlabeled data followed by independence tests with the "probabilistic labels" generated by EM, to obtain a new structure. EM is used again in the new structure and the cycle is repeated, until two subsequent networks are identical. It should be noted that such a scheme, however intuitively reasonable, has no convergence guarantees; one test even displayed oscillating behavior.

A second class of structure learning algorithms are score-based methods. At the heart of most score based methods is the likelihood of the training data, with penalty terms to avoid overfitting. A good comparison of the different methods is found in [47]. Most existing methods cannot, in their present form, handle missing data in general and unlabeled data in particular. The structural EM (SEM) algorithm [48] is one attempt to learn structure with missing data. The algorithm attempts to maximize the Bayesian score using an EM-like scheme in the space of structures and parameters; the method performs an always-increasing search in the space of structures, but does not guarantee the attainment of even a local maximum. When learning the structure of a classifier, score based structure learning approaches have been strongly criticized. The problem 
is that with finite amounts of data, the a-posteriori probability of the class variable can have a small effect on the score, that is dominated by the marginal of the observables, therefore leading to poor classifiers $[11,12]$. Friedman et al. [11] showed that TAN surpasses score-based methods for the fully labeled case, when learning classifiers. The point is that with unlabeled data, scorebased methods, such as SEM, are likely to go astray even more than it has been reported in the supervised case; the marginal of the observables further dominates the likelihood portion of the score as the ratio of unlabeled data increases.

\section{B. Classification driven stochastic structure search (SSS)}

Both the score-based and independence-based methods try to find the correct structure of the Bayesian network, but fail to do so because there is not enough data for either reliable independence tests or for a search that yields a good classifier. Consider the following alternative. As we are interested in finding a structure that performs well as a classifier, it would be natural to design algorithms that use classification error as the guide for structure learning. Here we can further leverage on the properties of semi-supervised learning: we know that unlabeled data can indicate incorrect structure through degradation of classification performance, and we also know that classification performance improves with the correct structure. Thus, a structure with higher classification accuracy over another indicates an improvement towards finding the optimal classifier.

To learn the structure using classification error, we must adopt a strategy for searching through the space of all structures in an efficient manner while avoiding local maxima. In this section, we propose a method that can effectively search for better structures with an explicit focus on classification. We essentially need to find a search strategy that can efficiently search through the space of structures. As we have no simple closed-form expression that relates structure with classification error, it would be difficult to design a gradient descent algorithm or a similar iterative method. Even if we did that, a gradient search algorithm would be likely to find a local minimum because of the size of the search space.

First we define a measure over the space of structures which we want to maximize:

Definition 1: The inverse error measure for structure $S^{\prime}$ is

$$
\operatorname{inv}_{e}\left(S^{\prime}\right)=\frac{\frac{1}{p_{S^{\prime}}(\hat{c}(X) \neq C)}}{\sum_{S} \frac{1}{p_{S}(\hat{c}(X) \neq C)}},
$$


where the summation is over the space of possible structures and $p_{S}(\hat{c}(X) \neq C)$ is the probability of error of the best classifier learned with structure $S$.

We use Metropolis-Hastings sampling [49] to generate samples from the inverse error measure, without having to ever compute it for all possible structures. For constructing the MetropolisHastings sampling, we define a neighborhood of a structure as the set of directed acyclic graphs to which we can transit in the next step. Transition is done using a predefined set of possible changes to the structure; at each transition a change consists of a single edge addition, removal or reversal. We define the acceptance probability of a candidate structure, $S_{n e w}$, to replace a previous structure, $S_{t}$ as follows:

$$
\min \left(1,\left(\frac{i n v_{e}\left(S^{\text {new }}\right)}{i n v_{e}\left(S^{t}\right)}\right)^{1 / T} \frac{q\left(S^{t} \mid S^{\text {new }}\right)}{q\left(S^{\text {new }} \mid S^{t}\right)}\right)=\min \left(1,\left(\frac{p_{\text {error }}^{t}}{p_{\text {error }}^{\text {new }}}\right)^{1 / T} \frac{N_{t}}{N_{\text {new }}}\right)
$$

where $q\left(S^{\prime} \mid S\right)$ is the transition probability from $S$ to $S^{\prime}$ and $N_{t}$ and $N_{\text {new }}$ are the sizes of the neighborhoods of $S_{t}$ and $S_{\text {new }}$ respectively; this choice corresponds to equal probability of transition to each member in the neighborhood of a structure. This choice of neighborhood and transition probability creates a Markov chain which is aperiodic and irreducible, thus satisfying the Markov chain Monte Carlo (MCMC) conditions [50]. We summarize the algorithm, which we name Stochastic Structure Search (SSS), in Figure 4.

We add $T$ as a temperature factor in the acceptance probability. Roughly speaking, $T$ close to 1 would allow acceptance of more structures with higher probability of error than previous structures. $T$ close to 0 mostly allows acceptance of structures that improve probability of error. A fixed $T$ amounts to changing the distribution being sampled by the MCMC, while a decreasing $T$ is a simulated annealing run, aimed at finding the maximum of the inverse error measures. The rate of decrease of the temperature determines the rate of convergence. Asymptotically in the number of data, a logarithmic decrease of $T$ guarantees convergence to a global maximum with probability that tends to one [51].

The SSS algorithm, with a logarithmic cooling schedule $T$, can find a structure that is close to minimum probability of error. There are two caveats though. First, the logarithmic cooling schedule is very slow. We use faster cooling schedules and a starting point which is the best out of either the NB classifier or the TAN classifier. Second, we never have access to the true probability of error for any given structure, $p_{\text {error }}^{S}$. Instead, we use the empirical error over the training data (denoted as $\hat{p}_{\text {error }}^{S}$ ). 
Procedure Stochastic structure search (SSS):

- Fix the network structure to some initial structure, $S_{0}$.

- Estimate the parameters of the structure $S_{0}$ and compute the probability of error $p_{\text {error }}^{0}$.

- Set $t=0$.

- Repeat, until a maximum number of iterations is reached (MaxIter),

- Sample a new structure $S_{\text {new }}$, from the neighborhood of $S_{t}$ uniformly, with probability $1 / N_{t}$.

- Learn the parameters of the new structure using maximum likelihood estimation. Compute the probability of error of the new classifier, $p_{\text {error }}^{\text {new }}$.

- Accept $S_{\text {new }}$ with probability given in Eq.(8).

- If $S_{\text {new }}$ is accepted, set $S_{t+1}=S_{\text {new }}$ and $p_{\text {error }}^{t+1}=p_{\text {error }}^{\text {new }}$ and change $T$ according to the temperature decrease schedule. Otherwise $S_{t+1}=S_{t}$.

$-t=t+1$.

- Return the structure $S_{j}$, such that $j=\arg \min _{0 \leq j \leq \text { MaxIter }}\left(p_{\text {error }}^{j}\right)$.

Fig. 4. Stochastic structure search algorithm

To avoid the problem of overfitting several approaches are possible. The first is cross-validation; the labeled training data is split to smaller sets and several tests are performed using the smaller sets as test sets. However, this approach can significantly slow down the search, and is suitable only if the labeled training set is moderately large. Another approach is to penalize different structures according to some complexity measure. We could use the BIC or MDL complexity measure, but we chose to use the multiplicative penalty term derived from structural risk minimization since it is directly related to the relationship between training error and generalization error. We define a modified error term for use in Eq. (7) and (8):

$$
\left(\hat{p}_{\text {error }}^{S}\right)^{\text {mod }}=\frac{\hat{p}_{\text {error }}^{S}}{1-c \cdot \sqrt{\frac{h_{S}\left(\log \frac{2 n}{h_{S}}+1\right)-\log (\eta / 4)}{n}}},
$$

where $h_{S}$ is the Vapnik-Chervonenkis (VC) dimension of the classifier with structure $S, n$ is the number of training records, $\eta$ and $c$ are between 0 and 1 .

To approximate the VC dimension, we use $h_{S} \propto N_{S}$, where $N_{S}$ is the number of (free) 
parameters in the Markov blanket of the class variable in the network, assuming that all variables are discrete. We point the reader to [52], in which it was shown that the VC dimension of a Naive Bayes classifier is linearly proportional to the number of parameters. It is possible to extend this result to networks where the features are all descendants of the class variable. For more general networks, features that are not in the Markov blanket of the class variable cannot effect its value in classification (assuming there are no missing values for any feature), justifying the above approximation. In our initial experiments, we found that the multiplicative penalty outperformed the holdout method and the MDL and BIC complexity measures.

\section{Evaluation using UCI machine learning datasets}

To evaluate structure learning methods with labeled and unlabeled data, we started with an empirical study involving simulated data. We artificially generated data to investigate: (1) whether the SSS algorithm finds a structure that is close to the structure that generated the data, and (2) whether the algorithm uses unlabeled data to improve the classification performance. A typical result is as follows. We generated data from a TAN structure with 10 features. The dataset consisted of 300 labeled and 30000 unlabeled records. We first estimated the Bayes error rate by learning with the correct structure and with a very large fully labeled dataset. We obtained a classification accuracy of $92.49 \%$. We learned one Naive Bayes classifier only with the labeled records, and another with both labeled and unlabeled records; likewise, we learned a TAN classifier only with the labeled records, and another with both labeled and unlabeled records, using the EM-TAN algorithm; and finally, we learned a Bayesian network classifier with our SSS algorithm using both labeled and unlabeled records. The results are presented in the first row of Table I. With the correct structure, adding unlabeled data improves performance significantly (columns TAN-L and EM-TAN). Note that adding unlabeled data degraded the performance from $16 \%$ error to $40 \%$ error when we learned the Naive Bayes classifier. The structure search algorithm comes close to the performance of the classifier learned with the correct structure. Figure 5(a) shows the changes in the test and train error during the search process. The graph shows the first 600 moves of the search, initialized with the Naive Bayes structure. The error usually decreases as new structures are accepted; occasionally we see an increase in the error allowed by Metropolis-Hastings sampling.

Next, we performed experiments with some of the UCI datasets, using relatively small labeled 
TABLE I

Classification Results (IN \%) FOR NAIVE BAYES,TAN, EM-CBL1 AND STOCHASTIC STRUCTURE SEARCH. XX-L

INDICATES LEARNING ONLY WITH THE AVAILABLE LABELED DATA.

\begin{tabular}{|c|c|c|c|c|c|c|c|c|c|}
\hline \multirow[t]{2}{*}{ Dataset } & \multicolumn{2}{|c|}{ Train } & \multirow[t]{2}{*}{ Test } & \multirow[t]{2}{*}{ NB-L } & \multirow{2}{*}{$\begin{array}{l}\text { EM- } \\
\text { NB }\end{array}$} & \multirow[t]{2}{*}{ TAN-L } & \multirow{2}{*}{$\begin{array}{l}\text { EM- } \\
\text { TAN }\end{array}$} & \multirow{2}{*}{$\begin{array}{l}\text { EM- } \\
\text { CBL1 }\end{array}$} & \multirow[t]{2}{*}{ SSS } \\
\hline & \# lab & \# unlab & & & & & & & \\
\hline $\begin{array}{l}\text { TAN } \\
\text { artificial }\end{array}$ & 300 & 30000 & 50000 & $83.4 \pm 0.2$ & $59.2 \pm 0.2$ & $90.9 \pm 0.1$ & $91.9 \pm 0.1$ & N/A & $91.1 \pm 0.1$ \\
\hline Satimage & 600 & 3835 & 2000 & $81.7 \pm 0.9$ & $77.5 \pm 0.9$ & $\mathbf{8 3 . 5} \pm \mathbf{0 . 8}$ & $81.0 \pm 0.9$ & $\mathbf{8 3 . 5} \pm \mathbf{0 . 8}$ & $83.4 \pm 0.8$ \\
\hline Shuttle & 100 & 43400 & 14500 & $82.4 \pm 0.3$ & $76.1 \pm 0.4$ & $81.2 \pm 0.3$ & $90.5 \pm 0.2$ & $91.8 \pm 0.2$ & $96.3 \pm 0.2$ \\
\hline Adult & 6000 & 24163 & 15060 & $83.9 \pm 0.3$ & $73.1 \pm 0.4$ & $84.7 \pm 0.3$ & $80.0 \pm 0.3$ & $82.7 \pm 0.3$ & $85.0 \pm 0.3$ \\
\hline Chess & 150 & 1980 & 1060 & $79.8 \pm 1.2$ & $62.1 \pm 1.5$ & $\mathbf{8 7 . 0} \pm \mathbf{1 . 0}$ & $71.2 \pm 1.4$ & $81.0 \pm 1.2$ & $76.0 \pm 1.3$ \\
\hline
\end{tabular}

sets and large unlabeled sets (Table I). The results suggest that structure learning holds the most promise in utilizing the unlabeled data. There is no clear 'winner' approach, although SSS yields better results in most cases. We see performance degradation with NB for every dataset. EMTAN can sometimes improve performance over TAN with just labeled data (Shuttle). With the Chess dataset, discarding the unlabeled data and using only TAN seems the best approach. We have compared two likelihood based structure learning methods (K2 and MCMC) on the same datasets as well [34], showing that even if we allow the algorithms to use large labeled datasets to learn the structure, the resultant networks still suffer from performance degradation when learned with unlabeled data.

Illustrating the iterations of the SSS algorithm, Figure 5(b) shows the changes in error for the shuttle dataset.

\section{LEARning BAYESIAN NeTwORK Classifiers FOR HCI APPliCATIONS}

The experiments in the previous section discussed commonly used machine learning datasets. In the next sections we discuss two HCI applications that could benefit from the use of unlabeled data. We start with facial expression recognition. 


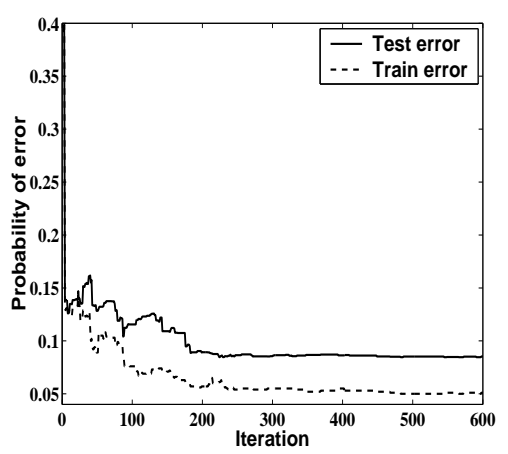

(a)

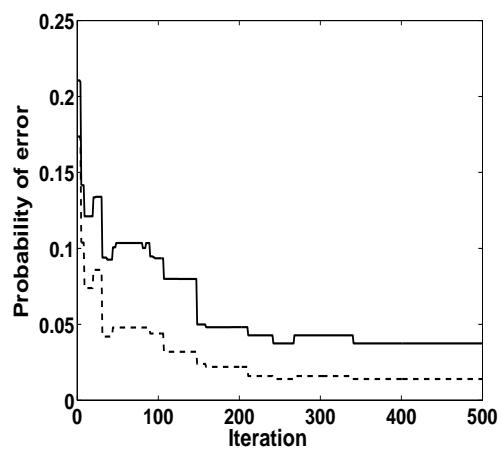

(b)

Fig. 5. Train and test error during the structure search for the artificial data (a) and shuttle data (b) for the labeled and unlabeled data experiments.

\section{A. Facial Expression Recognition using Bayesian Network Classifiers}

Since the early 1970s, Paul Ekman and his colleagues have performed extensive studies of human facial expressions [53] and found evidence to support universality in facial expressions. These "universal facial expressions" are those representing happiness, sadness, anger, fear, surprise, and disgust. Ekman's work inspired many researchers to analyze facial expressions by means of image and video processing. By tracking facial features and measuring the amount of facial movement, they attempt to categorize different facial expressions. Recent work on facial expression analysis and recognition has used these "basic expressions" or a subset of them. In [54], Pantic and Rothkrantz provide an in depth review of much of the research done in automatic facial expression recognition in recent years.

One of the challenges facing researchers attempting to design facial expression recognition systems is the relatively small amount of available labeled data. Construction and labeling of a good database of images or videos of facial expressions requires expertise, time, and training of subjects. Only a few such databases are available, such as the Cohn-Kanade database [55]. However, collecting, without labeling, data of humans displaying expressions is not as difficult. Therefore, it is beneficial to use classifiers that can be learned with a combination of some labeled data and a large amount of unlabeled data. As such we use (generative) Bayesian network classifiers.

We have developed a real time facial expression recognition system [56]. The system uses a model based non-rigid face tracking algorithm [57] to extract motion features (seen in Figure 7) 
that serve as input to a Bayesian network classifier used for recognizing the different facial expressions. There are two main motivations for using Bayesian network classifiers in this problem. The first is the ability to learn with unlabeled data and infer the class label even when some of the features are missing (e.g., due to failure in tracking because of occlusion). The second motivation is that it is possible to extend the system to fuse other modalities, such as audio, in a principled way by simply adding subnetworks representing the audio features.

1) Experimental Design: We use two different databases, a database collected by Chen and Huang [58] and the Cohn-Kanade AU code facial expression database [55]. The first is a database of subjects that were instructed to display facial expressions corresponding to the six types of emotions. All the tests of the algorithms are performed on a set of five people, each one displaying six sequences of each one of the six emotions, starting and ending at the neutral expression. The video sampling rate was $30 \mathrm{~Hz}$, and a typical emotion sequence is about 70 samples long ( $\sim 2 s$ ). Figure 6(upper row) shows one frame of each subject.

The Cohn-Kanade database [55] consists of expression sequences of subjects, starting from a neutral expression and ending in the peak of the facial expression. There are 104 subjects in the database. Because for some of the subjects, not all of the six facial expressions sequences were available to us, we used a subset of 53 subjects, for which at least four of the sequences were available. For each subject there is at most one sequence per expression with an average of 8 frames for each expression. Figure 6(lower row) shows some examples used in the experiments. A summary of both databases is presented in Table II. We measure the accuracy with respect to the classification result of each frame, where each frame in the video sequence was manually labeled to one of the expressions (including neutral). This manual labeling can introduce some 'noise' in our classification because the boundary between neutral and the expression of a sequence is not necessarily optimal, and frames near this boundary might cause confusion between the expression and the neutral.

2) Experimental results with labeled data: We start with experiments using all our labeled data. This can be viewed as an upper bound on the performance of the classifiers trained with most of the labels removed. For the labeled only case, we also compare results with training of an artificial Neural network (ANN) so as to test how Bayesian network classifiers compare with a different kind of classifier for this problem. We perform person independent tests by partitioning the data such that the sequences of some subjects are used as the test sequences 


\begin{tabular}{|c|c|c|c|c|}
\hline Database & \# of Subjects & $\begin{array}{c}\text { Overall \# of sequences } \\
\text { per expression }\end{array}$ & $\begin{array}{c}\text { \# of sequences per subject } \\
\text { per expression }\end{array}$ & $\begin{array}{c}\text { average \# of frames } \\
\text { per expression }\end{array}$ \\
\hline \hline Chen-Huang DB & 5 & 30 & 6 & 70 \\
\hline Cohn-Kanade DB & 53 & 53 & 1 & 8 \\
\hline
\end{tabular}
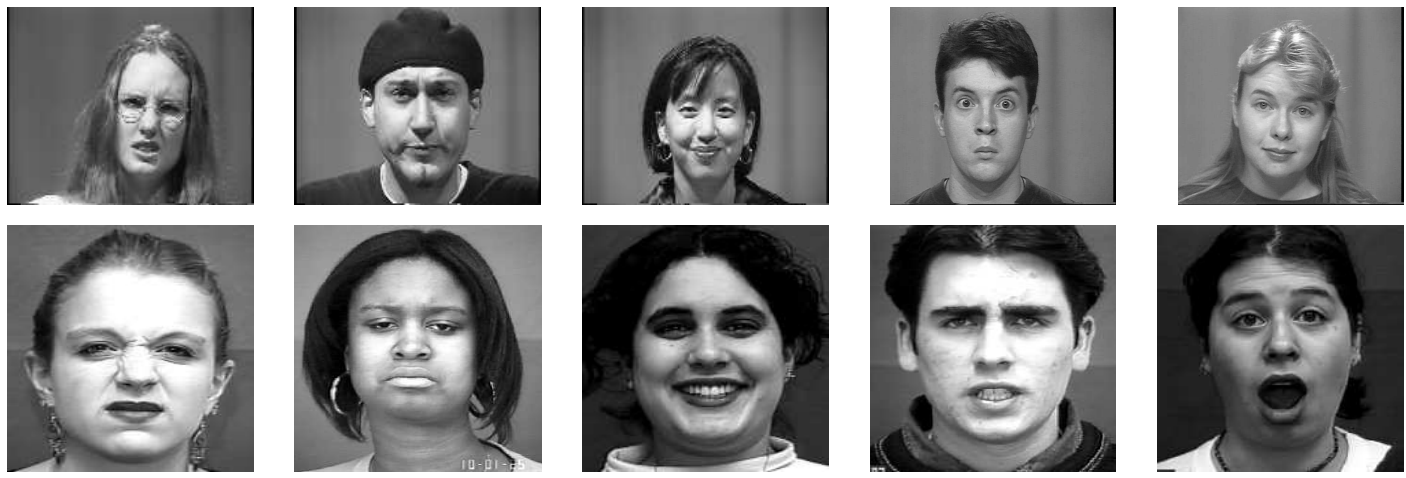

Fig. 6. Examples of images from the video sequences used in the experiment. Top row shows subjects from the Chen-Huang DB, bottom row shows subjects from the Cohn-Kanade DB (printed with permission from the researchers).

and the sequences of the remaining subjects are used as training sequences. Table III shows the recognition rate of the test for all classifiers. The classifier learned with the SSS algorithm outperforms both the NB and TAN classifiers, while ANN does not perform well compared to all the others.

3) Experiments with labeled and unlabeled data: We perform person-independent experiments with labeled and unlabeled data. We first partition the data to a training set and a test set $(2 / 3$ training, 1/3 for testing), and choose by random a portion of the training set and remove the labels. This procedure ensures that the distribution of the labeled and the unlabeled sets are the same.

We then train Naive Bayes and TAN classifiers, using just the labeled part of the training data and the combination of labeled and unlabeled data. We also use the SSS and the EM-CBL1 algorithms to train a classifier using both labeled and unlabeled data (we do not search for the 


\begin{tabular}{|c|c|c|c|c|}
\hline & NB & TAN & SSS & ANN \\
\hline Chen-Huang Database & 71.78 & 80.31 & $\underline{83.62}$ & 66.44 \\
\hline Cohn-Kandade Database & 77.70 & 80.40 & $\underline{81.80}$ & 73.81 \\
\hline
\end{tabular}

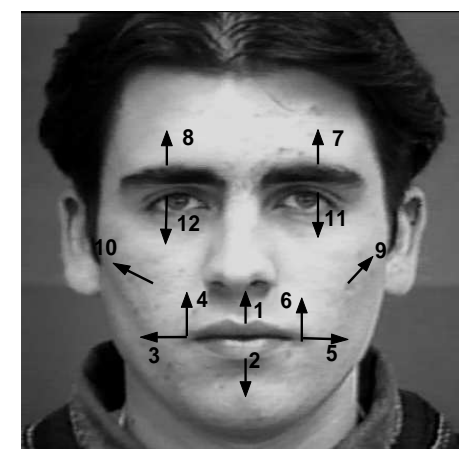

Fig. 7. Motion units extracted from face tracking.

structure with just the labeled part because it is too small for performing a full structure search).

Table IV shows the results of the experiments. We see that with NB and TAN, when using 200 and 300 labeled samples, adding the unlabeled data degrades the performance of the classifiers, and we would have been better off not using the unlabeled data. We also see that EM-CBL1 performs poorly in both cases. Using the SSS algorithm, we are able to improve the results and utilize the unlabeled data to achieve performance which is higher than using just the labeled data with NB and TAN. The fact that the performance is lower than in the case when all the training set was labeled (about $75 \%$ compared to over $80 \%$ ) implies that the relative value of labeled data is higher than of unlabeled data, as was shown by Castelli [26]. However, had there been more unlabeled data, the performance would be expected to improve.

\section{B. Applying Bayesian Network Classifiers to Face Detection}

We apply Bayesian network classifiers to the problem of face detection, with the purpose of showing that using our proposed methods, semi-supervised learning can be used to learn good face detectors. We take an appearance based approach, using the intensity of image pixels as the 


\begin{tabular}{|c|c|c|c|c|c|c|c|c|c|}
\hline \multirow[t]{2}{*}{ Dataset } & \multicolumn{2}{|c|}{ Train } & \multirow[t]{2}{*}{ Test } & \multirow[t]{2}{*}{ NB-L } & \multirow{2}{*}{$\begin{array}{l}\text { EM- } \\
\text { NB }\end{array}$} & \multirow[t]{2}{*}{ TAN-L } & \multirow{2}{*}{$\begin{array}{l}\text { EM- } \\
\text { TAN }\end{array}$} & \multirow{2}{*}{$\begin{array}{l}\text { EM- } \\
\text { CBL1 }\end{array}$} & \multirow[t]{2}{*}{ SSS } \\
\hline & \# lab & \# unlab & & & & & & & \\
\hline Cohn-Kanade & 200 & 2980 & 1000 & $72.5 \pm 1.4$ & $69.1 \pm 1.4$ & $72.9 \pm 1.4$ & $69.3 \pm 1.4$ & $66.2 \pm 1.5$ & $74.8 \pm 1.4$ \\
\hline Chen-Huang & 300 & 11982 & 3555 & $71.3 \pm 0.8$ & $58.5 \pm 0.8$ & $72.5 \pm 0.7$ & $62.9 \pm 0.8$ & $65.9 \pm 0.8$ & $\mathbf{7 5 . 0} \pm \mathbf{0 . 7}$ \\
\hline
\end{tabular}

features for the classifier. For learning and defining the Bayesian network classifiers, we must look at fixed size windows and learn how a face appears in such windows, where we assume that the face appears in most of the window's pixels. The goal of the classifier would be to determine if the pixels in a fixed size window are those of a face or non-face.

We note that there have been numerous appearance based approaches for face detection, many with considerable success (see Yang et al. [59] for a detailed review on the state-of the-art in face detection). However, there has not been any attempt, to our knowledge, to use semi-supervised learning in face detection. While labeled databases of face images are available, a universally robust face detector is still difficult to construct. The main challenge is that faces appear very different under different lighting conditions, expressions, with or without glasses, facial hair, makeup, etc. A classifier trained with some labeled images and a large number of unlabeled images would enable incorporating many more facial variations without the need to label huge datasets.

In our experiments we used a training set consisting of 2429 faces and 10000 non faces obtained from the MIT CBCL Face database \#1 [60]. Each face image is cropped and resampled to a $8 \times 8$ window, thus we have a classifier with 64 features. We also randomly rotate and translate the face images to create a training set of 10000 face images. In addition we have available 10000 non-face images. We leave out 1000 images (faces and non-faces) for testing and train the Bayesian network classifier on the remaining 19000. In all the experiments we learn a Naive Bayes, a TAN, and two general generative Bayesian network classifiers, the latter using the EM-CBL1 and the SSS algorithms.

To compare the results of the classifiers, we use the receiving operating characteristic (ROC) 


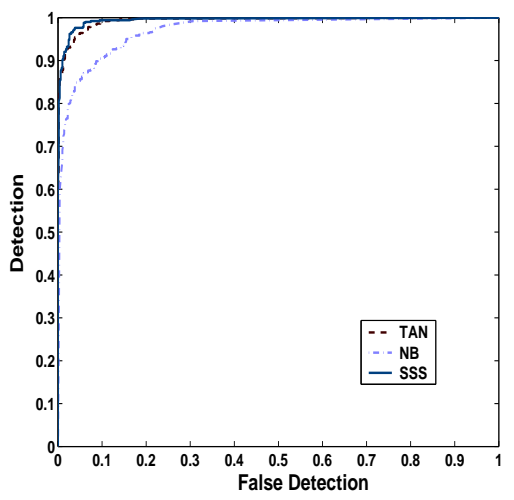

(a)

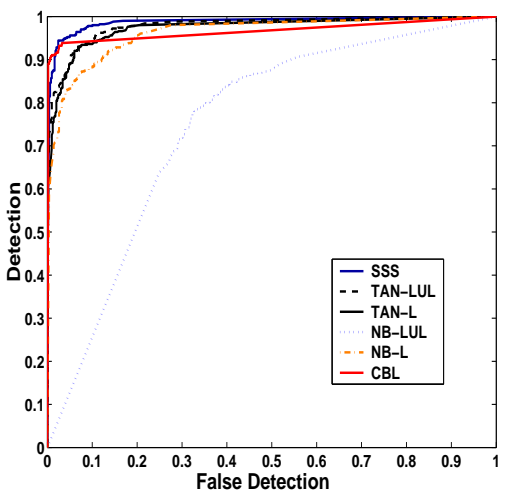

(b)

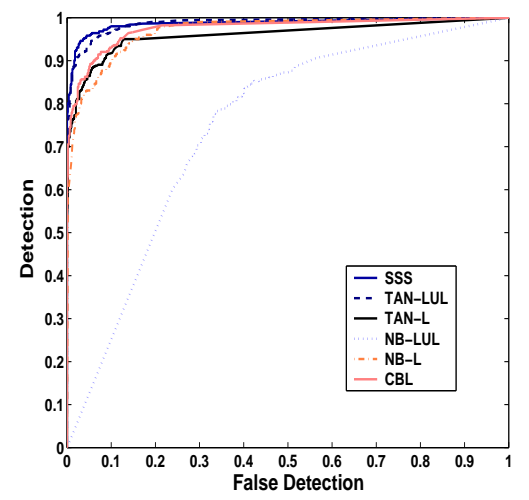

(c)

Fig. 8. ROC curves showing detection rates of faces compared to false detection of faces of the different (SSS, TAN and NB) classifiers and different ratios of labeled and unlabeled data, (a) with all the data labeled (no unlabeled data), (b) with $95 \%$ of the data unlabeled, (c) with $97.5 \%$ of the data unlabeled.

curves. The ROC curves show, under different classification thresholds, ranging from 0 to 1 , the probability of detecting a face in a face image, $P_{D}=P(\hat{C}=$ face $\mid C=$ face $)$, against the probability of falsely detecting a face in a non-face image, $P_{F D}=P(\hat{C}=$ face $\mid C \neq$ face $)$.

We first learn using all the training data being labeled. Figure 8(a) shows the resultant ROC curve for this case. The classifier learned with the SSS algorithm outperforms both TAN and NB classifiers, and all perform quite well, achieving about $96 \%$ detection rates with a low rate of false alarm.

Next we remove the labels of $95 \%$ of the training data (leaving only 475 labeled images) and train the classifiers. Figure 8(b) shows the resultant ROC curve for this case. We see that NB classifier using both labeled and unlabeled data performs very poorly. The TAN based on the 475 labeled images and the TAN based on the labeled and unlabeled images are close in performance, thus there was no significant degradation of performance when adding the unlabeled data. The classifier using all data and the SSS outperforms the rest with an ROC curve close to the best ROC curve in Figure 8(a). Figure 8(c) shows the ROC curve with only 250 labeled data used. Again, NB with both labeled and unlabeled performs poorly, while SSS outperforms the other classifiers with no great reduction of performance compared to the two other ROC curves. The experiment shows that using structure search, the unlabeled data was utilized successfully to achieve a classifier almost as good as if all the data was labeled. 


\section{SUMmary AND DisCUSSION}

Using unlabeled data to enhance the performance of classifiers trained with few labeled data has many applications in pattern recognition, computer vision, HCII, data mining, text recognition and more. To fully utilize the potential of unlabeled data, the abilities and limitations of existing methods must be understood.

The main contributions of this paper can be summarized as follows:

1) We have derived and studied the asymptotic behavior of semi-supervised learning based on maximum likelihood estimation. We presented a detailed analysis of performance degradation from unlabeled data, showing that it is directly related to modeling assumptions, regardless of numerical instabilities or finite sample effects.

2) We discussed the implications of the analysis of semi-supervised learning on Bayesian network classifiers; namely the importance of structure when unlabeled data are used in training. We listed the possible shortcomings of likelihood-based structural learning algorithms when learning classifiers, especially when unlabeled data are present.

3) We introduced a classification driven structure search algorithm based on MetropolisHastings sampling, and showed that it performs well both on fully labeled datasets and on labeled and unlabeled training sets. As a note for practitioners, the SSS algorithm appears to work well for relatively large datasets and difficult classification problems that are represented by complex structures. Large datasets are those where there are enough labeled data for reliable estimation of the empirical error, allowing search for complex structures, and there are enough unlabeled data to reduce the estimation variance of complex structures.

4) We presented our real-time facial expression recognition system using a model-based face tracking algorithm and Bayesian network classifiers. We showed experiments using both labeled and unlabeled data.

5) We presented the use of Bayesian network classifiers for learning to detect faces in images. We note that while finding a good classifier is a major part of any face detection system, there are many more components that need to be designed for such a system to work on natural images (e.g., ability to detect at multi-scales, highly varying illumination, large rotations of faces and partial occlusions). Our goal was to present the first step in designing 
such a system and show the feasibility of the approach when training with labeled and unlabeled data.

Our discussion of semi-supervised learning for Bayesian networks suggests the following path: when faced with the option of learning Bayesian networks with labeled and unlabeled data, start with Naive Bayes and TAN classifiers, learn with only labeled data and test whether the model is correct by learning with the unlabeled data. If the result is not satisfactory, then SSS can be used to attempt to further improve performance with enough computational resources. If none of the methods using the unlabeled data improve performance over the supervised TAN (or Naive Bayes), either discard the unlabeled data or try to label more data, using active learning for example.

Following our investigation of semi-supervised learning, there are several important open theoretical questions and research directions:

- Is it possible to find necessary and sufficient conditions for performance degradation to occur? Finding such conditions are of great practical significance. Knowing these conditions can lead to the design of new useful tests that will indicate when unlabeled can be used or when they should be discarded, or if a different model should be chosen.

- An important question is whether other semi-supervised learning methods, such as transductive SVM [61] or co-training [62], will exhibit the phenomenon of performance degradation? While no extensive studies have been performed, a few results from the literature suggest that it is a realistic conjecture. Zhang and Oles [2] demonstrated that transductive SVM can cause degradation of performance when unlabeled data are added. Ghani [63] described experiments where the same phenomenon occurred with co-training. If the causes of performance degradation are similar for different algorithms, it should be possible to present a unified theory for semi-supervised learning.

- Are there performance guarantees for semi-supervised learning with finite amounts of data, labeled and unlabeled? In supervised learning such guarantees are studied extensively. PAC and risk minimization bounds help in determining the minimum amount of (labeled) data necessary to learn a classifier with good generalization performance. However, there are no existing bounds on the classification performance when training with labeled and unlabeled data. Finding such bounds can be derived using principles in estimation theory, based on 
asymptotic covariance properties of the estimator. Other bounds can be derived using PAC theoretical approaches. Existence of such bounds can immediately lead to new algorithms and approaches, better utilizing unlabeled data.

- Can we use the fact that unlabeled data indicate model incorrectness to actively learn better models? The use of active learning seems promising whenever possible, and it might be possible to extend active learning to learn better models, not just enhancement of the parameter estimation.

In closing, this work should be viewed as a combination of three main components. The theory showing the limitations of unlabeled data is used to motivate the design of algorithms to search for better performing structures of Bayesian networks and finally, the successful application to the real-world problems we were interested in solving by learning with labeled and unlabeled data.

\section{ACKNOWLEDGMENTS}

The authors would like to acknowledge Alex Bronstein and Marsha Duro of HP-Labs for their support and discussions on the analysis of semi-supervised learning. Portions of this work have been done in HP-Labs, Palo-Alto. Ira Cohen has been funded by an HP Fellowship. Financial support has also been granted by HP-Brazil through the Eldorado Research Institute. The authors would like to thank Jilin Tu for his work on the face tracking, Roy Wang and Ming-Hsuan Yang for their help with the face detection database and Jeffery Cohn and Larry Chen for the facial expression databases. We would like to thank Moises Goldszmidt, Ashutosh Garg, Ismênia Blavatsky, and Michael Lew for discussions on various parts of this work and the reviewers for their helpful comments. We coded our own Naive Bayes and TAN classifiers in the Java language, using the libraries of the JavaBayes system (freely available at http://www.cs.cmu.edu/ javabayes).

\section{REFERENCES}

[1] B. Shahshahani and D. Landgrebe, "Effect of unlabeled samples in reducing the small sample size problem and mitigating the Hughes phenomenon," IEEE Transactions on Geoscience and Remote Sensing, vol. 32, no. 5, pp. 1087-1095, 1994.

[2] T. Zhang and F. Oles, "A probability analysis on the value of unlabeled data for classification problems," in International Conference on Machine Learning (ICML), pp. 1191-1198, 2000.

[3] K. Nigam, A. McCallum, S. Thrun, and T. Mitchell, "Text classification from labeled and unlabeled documents using EM," Machine Learning, vol. 39, no. 2, pp. 103-134, 2000. 
[4] R. Bruce, "Semi-supervised learning using prior probabilities and EM." presented at the International Joint Conference of AI Workshop on Text Learning: Beyond Supervision, Seattle, Washington, 2001.

[5] S. Baluja, "Probabilistic modelling for face orientation discrimination: Learning from labeled and unlabeled data," in Neural Information and Processing Systems (NIPS), pp. 854-860, 1998.

[6] R. Kohavi, "Scaling up the accuracy of naive Bayes classifiers: A decision-tree hybrid," in Proc. Second Int. Conference on Knowledge Discovery and Data Mining, pp. 202-207, 1996.

[7] I. Cohen, F. G. Cozman, and A. Bronstein, "On the value of unlabeled data in semi-supervised learning based on maximumlikelihood estimation,” Tech. Rep. HPL-2002-140, HP-Labs, 2002.

[8] J. Pearl, Probabilistic Reasoning in Intelligent Systems: Networks of Plausible Inference. San Mateo, California: Morgan Kaufmann, 1988.

[9] A. Garg, V. Pavlovic, and J. Rehg, "Boosted learning in dynamic bayesian networks for multimodal speaker detection," Proceedings of the IEEE, vol. 91, pp. 1355 - 1369, Sep 2003.

[10] N. Oliver, E. Horvitz, and A. Garg, "Hierarchical representations for learning and inferring office activity from multimodal information," in International Conference on Multimodal Interfaces, (ICMI), 2002.

[11] N. Friedman, D. Geiger, and M. Goldszmidt, "Bayesian network classifiers," Machine Learning, vol. 29, no. 2, pp. 131-163, 1997.

[12] R. Greiner and W. Zhou, "Structural extension to logistic regression: Discriminative parameter learning of belief net classifiers," in Proc. of the Annual National Conference on Artificial Intelligence (AAAI), pp. 167-173, 2002.

[13] P. Ekman and W. Friesen, Facial Action Coding System: Investigator's Guide. Palo Alto: Consulting Psychologists Press, 1978.

[14] C. L. Blake and C. J. Merz, "UCI repository of machine learning databases, University of California, Irvine, Dept. of Information and Computer Sciences," 1998.

[15] L. Devroye, L. Gyorfi, and G. Lugosi, A Probabilistic Theory of Pattern Recognition. New York: Springer Verlag, 1996.

[16] A. Corduneanu and T. Jaakkola, "Continuations methods for mixing heterogeneous sources," in Uncertainty in Artificial Intelligence (UAI), pp. 111-118, 2002.

[17] R. Chhikara and J. McKeon, "Linear discriminant analysis with misallocation in training samples," Journal of the American Statistical Association, vol. 79, pp. 899-906, 1984.

[18] C. Chittineni, "Learning with imperfectly labeled examples," Pattern recognition, vol. 12, pp. 271-281, 1981.

[19] T. Krishnan and S. Nandy, "Efficiency of discriminant analysis when initial samples are classified stochastically," Pattern recognition, vol. 23, pp. 529-537, 1990.

[20] T. Krishnan and S. Nandy, "Efficiency of logistic-normal supervision," Pattern recognition, vol. 23, pp. 1275-1279, 1990.

[21] S. Pal and e. A. Pal, Pattern Recognition from classical to modern approaches. New Jersey: World Scientific, 2002.

[22] D. B. Cooper and J. H. Freeman, "On the asymptotic improvement in the outcome of supervised learning provided by additional nonsupervised learning," IEEE Transactions on Computers, vol. C-19, pp. 1055-1063, November 1970.

[23] D. W. Hosmer, "A comparison of iterative maximum likelihood estimates of the parameters of a mixture of two normal distributions under three different types of sample," Biometrics, vol. 29, pp. 761-770, December 1973.

[24] T. J. O'Neill, "Normal discrimination with unclassified observations," Journal of the American Statistical Association, vol. 73, no. 364, pp. 821-826, 1978.

[25] S. Ganesalingam and G. J. McLachlan, "The efficiency of a linear discriminant function based on unclassified initial samples," Biometrika, vol. 65, pp. 658-662, December 1978. 
[26] V. Castelli, The relative value of labeled and unlabeled samples in pattern recognition. $\mathrm{PhD}$ thesis, Stanford University, Palo Alto, CA, 1994.

[27] J. Ratsaby and S. S. Venkatesh, "Learning from a mixture of labeled and unlabeled examples with parametric side information," in Proceedings of the Eigth Annual Conference on Computational Learning Theory, pp. 412-417, 1995.

[28] T. Mitchell, "The role of unlabeled data in supervised learning." presented at the Sixth International Colloquium on Cognitive Science, San Sebastian, Spain, 1999.

[29] D. J. Miller and H. S. Uyar, "A mixture of experts classifier with learning based on both labelled and unlabelled data," in Neural Information and Processing Systems (NIPS), pp. 571-577, 1996.

[30] M. Collins and Y. Singer, "Unupervised models for named entity classification," in International Conference on Machine Learning (ICML), pp. 327-334, 2000.

[31] F. DeComité, F. Denis, R. Gilleron, and F. Letouzey, "Positive and unlabeled examples help learning," in Proc. of 10th International Conference on Algorithmic Learning Theory (O. Watanabe and T. Yokomori, eds.), (Berlin), pp. 219-230, Springer-Verlag, 1999.

[32] S. Goldman and Y. Zhou, "Enhancing supervised learning with unlabeled data," in International Conference on Machine Learning (ICML), pp. 327-334, 2000.

[33] F. G. Cozman and I. Cohen, "Unlabeled data can degrade classification performance of generative classifiers," in Fifteenth International Florida Artificial Intelligence Society Conference, pp. 327-331, 2002.

[34] I. Cohen, Semisupervised learning of classifiers with application to human-computer interaction. PhD thesis, University of Illinois at Urbana-Champaign, 2003.

[35] F. G. Cozman, I. Cohen, and M. Cirelo, "Semi-supervised learning of mixture models," in International Conference on Machine Learning (ICML), pp. 99-106, 2003.

[36] A. P. Dempster, N. M. Laird, and D. B. Rubin, "Maximum likelihood from incomplete data via the EM algorithm," Journal of the Royal Statistical Society, Series B, vol. 39, no. 1, pp. 1-38, 1977.

[37] H. White, "Maximum likelihood estimation of misspecified models," Econometrica, vol. 50, pp. 1-25, January 1982.

[38] F. G. Cozman and I. Cohen, "The effect of modeling errors in semi-supervised learning of mixture models: How unlabeled data can degrade performance of generative classifiers," tech. rep., University of Sao Paulo, 2003. http://www.poli.usp.br/p/fabio.cozman/Publications/lul.ps.gz.

[39] S. W. Ahmed and P. A. Lachenbruch, "Discriminant analysis when scale contamination is present in the initial sample," in Classification and Clustering, (New York), pp. 331-353, Academic Press Inc., 1977.

[40] G. J. McLachlan, Discriminant Analysis and Statistical Pattern Recognition. New York: John Wiley and Sons Inc., 1992.

[41] J. H. Friedman, "On bias, variance, 0/1-loss, and the curse-of-dimensionality," Data Mining and Knowledge Discovery, vol. 1, no. 1, pp. 55-77, 1997.

[42] M. Meila, Learning with mixture of trees. PhD thesis, Massachusetts Institute of Technology, Boston, MA, 1999.

[43] P. Spirtes, C. Glymour, and R. Scheines, Causation, Prediction, and Search. Cambridge: MIT Press, 2nd ed., 2000.

[44] J. Pearl, Causality: Models, Reasoning, and Inference. Cambridge: Cambridge University Press, 2000.

[45] J. Cheng, R. Greiner, J. Kelly, D. A. Bell, and W. Liu, "Learning Bayesian networks from data: an information-theory based approach," Artificial Intelligence Journal, vol. 137, pp. 43-90, May 2002.

[46] J. Cheng and R. Greiner, “Comparing Bayesian network classifiers,” in Uncertainty in Artificial Intelligence (UAI), pp. 101$108,1999$. 
[47] T. V. Allen and R. Greiner, "A model selection criteria for learning belief nets: An empirical comparison," in International Conference on Machine Learning (ICML), pp. 1047-1054, 2000.

[48] N. Friedman, "The Bayesian structural EM algorithm," in Uncertainty in Artificial Intelligence (UAI), pp. 129-138, 1998.

[49] N. Metropolis, A. W. Rosenbluth, M. N. Rosenbluth, A. H. Teller, and E. Teller, "Equation of state calculation by fast computing machines,” Journal of Chemical Physics, vol. 21, pp. 1087-1092, 1953.

[50] D. Madigan and J. York, "Bayesian graphical models for discrete data," Int. Statistical Review, vol. 63, no. 2, pp. 215-232, 1995.

[51] B. Hajek, "Cooling schedules for optimal annealing," Mathematics of Operational Research, vol. 13, pp. 311-329, May 1988.

[52] D. Roth, "Learning in natural language," in International Joint Conference on Artificial Intelligence, pp. 898-904, 1999.

[53] P. Ekman, "Strong evidence for universals in facial expressions: A reply to Russell's mistaken critique," Psychological Bulletin, vol. 115, no. 2, pp. 268-287, 1994.

[54] M. Pantic and L. J. M. Rothkrantz, "Automatic analysis of facial expressions: The state of the art," IEEE Trans on PAMI, vol. 22, no. 12, pp. 1424-1445, 2000.

[55] T. Kanade, J. Cohn, and Y. Tian, "Comprehensive database for facial expression analysis," in Automatic Face and Gesture Recognition ( $F G^{\prime} 00$ ), pp. 46-53, 2000.

[56] I. Cohen, N. Sebe, A. Garg, and T. S. Huang, "Facial expression recognition from video sequences," in Proceedings of the International Conference on Multimedia and Expo (ICME), pp. 121-124, 2002.

[57] H. Tao and T. S. Huang, "Connected vibrations: A modal analysis approach to non-rigid motion tracking," in IEEE Conference on Computer Vision and Pattern Recognition, pp. 735-740, 1998.

[58] L. S. Chen, Joint processing of audio-visual information for the recognition of emotional expressions in human-computer interaction. PhD thesis, University of Illinois at Urbana-Champaign, Urbana, IL, 2000.

[59] M. H. Yang, D. Kriegman, and N. Ahuja, "Detecting faces in images: A survey," IEEE Trans on PAMI, vol. 24, no. 1, pp. 34-58, 2002.

[60] MIT CBCL Face Database \#1. MIT Center For Biological and Computation Learning: http://www.ai.mit.edu/projects/cbcl, 2002.

[61] K. Bennett and A. Demiriz, "Semi-supervised support vector machines," in Neural Information and Processing Systems (NIPS), pp. 368-374, 1998.

[62] A. Blum and T. Mitchell, "Combining labeled and unlabeled data with co-training," in Proceedings of the Eleventh Annual Conference on Computational Learning Theory, pp. 92-100, 1998.

[63] R. Ghani, "Combining labeled and unlabeled data for multiclass text categorization," in International Conference on Machine Learning (ICML), pp. 187-194, 2002. 


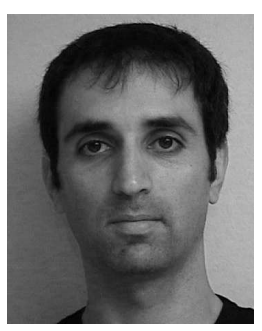

Ira Cohen earned his B.Sc. from Ben-Gurion University, Israel and his M.S. and PhD. from the University of Illinois at Urbana-Champaign in Electrical and Computer Engineering in 1998, 2000 and 2003 respectively. Since 2003 he is a research scientist at Hewlett-Packard research labs in Palo Alto, CA, where he works on machine learning theory with application to computer system and performance modeling. His research interests are in probabilistic models, computer vision, human computer interaction and system modeling.

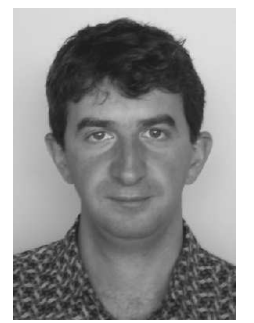

Nicu Sebe received his Ph.D. degree from Leiden University, The Netherlands in 2001. Currently, he is with the Faculty of Science, University of Amsterdam, The Netherlands, where he is doing research in the areas of multimedia information retrieval and human-computer interaction in computer vision applications. $\mathrm{He}$ is the author of the book Robust Computer Vision - Theory and Applications (Kluwer, April 2003) and of the upcoming book Computer Vision: A Machine Learning Approach (Kluwer, Summer 2004). He was the guest editor of a CVIU Special Issue on Video Retrieval and Summarization (December 2003), was the co-chair of the 5th ACM Multimedia Information Retrieval Workshop, MIR03 (in conjunction with ACM Multimedia 2003) and is the co-chair of the Human Computer Interaction Workshop, HCI04 (in conjunction with ECCV 2004). He also was the technical program chair for the International Conference on Image and Video Retrieval, CIVR 2003. He has published over 50 technical papers in the areas of computer vision, content-based retrieval, pattern recognition, and human-computer interaction and has served on the program committee of several conferences in these areas. He is a member of IEEE and ACM.

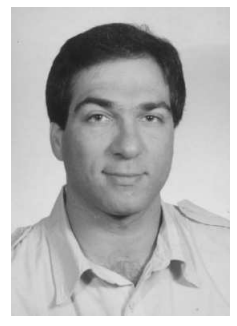

Fabio G. Cozman is an Associate Professor at the University of Sao Paulo, Brazil. He earned his PhD degree in Robotics in 1997 from the School of Computer Science, Carnegie Mellon University, and has worked on the theory and applications of sets of probability measures, Bayesian networks, and semisupervised learning. 


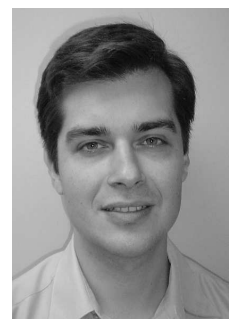

Marcelo C. Cirelo is an Electrical enginner and graduate student at Univ. of Sao Paulo, with interests in the areas of machine learning and computer vision - particularly in applications that require manipulation of labeled and unlabeled data.

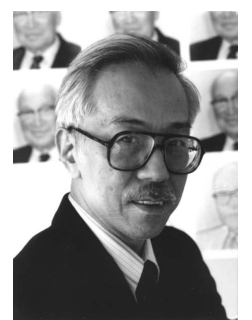

Thomas S. Huang received his B.S. Degree in Electrical Engineering from National Taiwan University, Taipei, Taiwan, China; and his M.S. and Sc.D. Degrees in Electrical Engineering from the Massachusetts Institute of Technology, Cambridge, Massachusetts. He was on the Faculty of the Department of Electrical Engineering at MIT from 1963 to 1973; and on the Faculty of the School of Electrical Engineering and Director of its Laboratory for Information and Signal Processing at Purdue University from 1973 to 1980. In 1980, he joined the University of Illinois at Urbana-Champaign, where he is now William L. Everitt Distinguished Professor of Electrical and Computer Engineering, and Research Professor at the Coordinated Science Laboratory, and Head of the Image Formation and Processing Group at the Beckman Institute for Advanced Science and Technology and Co-Chair of the Institute's major research theme Human Computer Intelligent Interaction. Dr. Huang's professional interests lie in the broad area of information technology, especially the transmission and processing of multidimensional signals. He has published 14 books, and over 500 papers in Network Theory, Digital Filtering, Image Processing, and Computer Vision. $\mathrm{He}$ is a Member of the National Academy of Engineering; a Foreign Member of the Chinese Academies of Engineering and Sciences; and a Fellow of the International Association of Pattern Recognition, IEEE, and the Optical Society of American; and has received a Guggenheim Fellowship, an A.V. Humboldt Foundation Senior U.S. Scientist Award, and a Fellowship from the Japan Association for the Promotion of Science. He received the IEEE Signal Processing Society's Technical Achievement Award in 1987, and the Society Award in 1991. He was awarded the IEEE Third Millennium Medal in 2000. Also in 2000, he received the Honda Lifetime Achievement Award for "contributions to motion analysis". In 2001, he received the IEEE Jack S. Kilby Medal. In 2002, he received the King-Sun Fu Prize, International Association of Pattern Recognition; and the Pan Wen-Yuan Outstanding Research Award. He is a Founding Editor of the International Journal Computer Vision, Graphics, and Image Processing; and Editor of the Springer Series in Information Sciences, published by Springer Verlag. 